
.

\title{
Tyrosine kinase inhibitor treatments in patients with metastatic thyroid carcinomas: a retrospective study of the TUTHYREF network
}

\author{
Marie-Hélène Massicotte ${ }^{1,2}$, Maryse Brassard ${ }^{3}$, Médéric Claude-Desroches $^{4}$, \\ Isabelle Borget ${ }^{5}$, Françoise Bonichon ${ }^{6}$, Anne-Laure Giraudet ${ }^{7}$, Christine Do Cao ${ }^{8}$, \\ Cécile N Chougnet ${ }^{1}$, Sophie Leboulleux ${ }^{1}$, Eric Baudin ${ }^{1}$, Martin Schlumberger' and \\ Christelle de la Fouchardière ${ }^{9}$ \\ ${ }^{1}$ Department of Nuclear Medicine and Endocrine Oncology, Institut Gustave Roussy, Université Paris-Sud, 114 Rue \\ Edouard Vaillant, 94805 Villejuif, France, ${ }^{2}$ Endocrinology Service, Department of Medicine, Centre Hospitalier \\ Universitaire de Sherbrooke, 3001 12e Avenue Nord, Sherbrooke, Québec, Canada J1H 5N3, ${ }^{3}$ Endocrinology Service, \\ Department of Medicine, Centre Hospitalier Universitaire de Québec, Université Laval, 1401 18e Rue, Québec City, \\ Québec, Canada G1J 1Z4, ${ }^{4}$ Department of Radiology, Institut Universitaire de Cardiologie et de Pneumologie de \\ Québec, 2725 Chemin Sainte-Foy, Québec City, Québec, Canada G1V 4G5, ${ }^{5}$ Department of Biostatistic and \\ Epidemiology, Institut Gustave Roussy, Université Paris-Sud, 114 Rue Edouard Vaillant, 94805 Villejuif, France, \\ ${ }^{6}$ Department of Nuclear Medicine, Institut Bergonié, 229 Cours de I'Argonne, 33000 Bordeaux, France, \\ ${ }^{7}$ Department of Nuclear Medicine, Institut Curie, Hôpital René Huguenin, 35 Rue Dailly, 92210 Saint-Cloud, France, \\ ${ }^{8}$ Department of Endocrinology, Hôpital Huriez, Centre Hospitalier Régional Universitaire de Lille, 2 Avenue Oscar \\ Lambret, 59037 Lille, France and ${ }^{9}$ Consortium Cancer Thyroïdien, Hospices Civils de Lyon-Centre Anti-Cancéreux \\ Léon-Bérard, 28 Rue Laennec, 69008 Lyon, France
}

\author{
Correspondence \\ should be addressed \\ to M-H Massicotte \\ Email \\ marie-helene.massicotte@ \\ usherbrooke.ca
}

\begin{abstract}
Objective: Tyrosine kinase inhibitors (TKIs) are used to treat patients with advanced thyroid cancers. We retrospectively investigated the efficacy of TKIs administered outside of clinical trials in metastatic sites or locally advanced thyroid cancer patients from five French oncology centers.

Design and methods: There were 62 patients (37 men, mean age: 61 years) treated with sorafenib (62\%), sunitinib (22\%), and vandetanib (16\%) outside of clinical trials; 22 had papillary, five had follicular, five had Hürthle cell, 13 had poorly differentiated, and 17 had medullary thyroid carcinoma (MTC). Thirty-three, 25, and four patients were treated with one, two, and three lines of TKIs respectively. Primary endpoints were objective tumor response rate and progression-free survival (PFS). Sequential treatments and tumor response according to metastatic sites were secondary endpoints.

Results: Among the 39 sorafenib and 12 sunitinib treatments in differentiated thyroid carcinoma (DTC) patients, partial response (PR) rate was 15 and $8 \%$ respectively. In the $11 \mathrm{MTC}$ patients treated with vandetanib, 36\% had PR. Median PFS was similar in second-line compared with first-line sorafenib or sunitinib therapy (6.7 vs 7.0 months) in DTC patients, but there was no PR with second- and third-line treatments. Bone and pleural lesions were the most refractory sites to treatment. Conclusions: This is the largest retrospective study evaluating TKI therapies outside of clinical trials. DTC patients treated with second-line therapy had stable disease as best response, but had a similar median PFS compared with the first-line treatment.
\end{abstract}

\section{Introduction}

Approximately $5-10 \%$ of patients with differentiated thyroid cancer (DTC) eventually develop metastatic disease, of whom two-thirds present with iodine refractory disease (1). Medullary thyroid carcinomas (MTCs) account for $5-8 \%$ of all thyroid cancers, and distant metastases that are present at diagnosis in $7-23 \%$ of patients represent the (c) 2014 European Society of Endocrinology Printed in Great Britain
Published by Bioscientifica Ltd. 
main cause of cancer-related death (2). These patients often suffer from a progressive course despite local treatment modalities such as radiofrequency ablation or external beam radiation therapy, and cytotoxic chemotherapies have shown disappointing results.

In recent years, several trials have demonstrated the efficacy of tyrosine kinase inhibitors (TKIs) in the treatment of metastatic or locally advanced thyroid carcinomas (3). In phase II clinical trials, a partial response (PR) rate of about 20\% was seen in DTC patients treated with sorafenib $(4,5)$ or with sunitinib $(6,7)$. In a phase III trial, sorafenib significantly improved the median progression-free survival (PFS) from 5.8 months in the placebo group to 10.8 months (hazard ratio $(\mathrm{HR})=0.587, P<0.0001)$ (8). Vandetanib also produced a significant prolongation of PFS in DTC patients $(\mathrm{HR}=0.63, P=0.008)$ (9). Sorafenib was approved for the treatment of refractory advanced DTC by the US Food and Drug Administration (FDA) in November 2013, but to date no TKI is approved by the European Medicine Agency (EMA) for DTC treatment.

Clinical benefit was also seen in MTC patients treated with sorafenib and sunitinib in phase II studies $(6,7,10)$, and a phase III study showed that vandetanib prolonged median PFS from 19.3 months to more than 30.5 months $(\mathrm{HR}=0.46, P<0.001)$ with a PR rate of $45 \%(11)$. This study led to approval of vandetanib for the treatment of advanced MTC by the FDA in April 2011 and by the EMA in February 2012. In a phase III trial, cabozantinib improved PFS from 4 to 11 months and PR was observed in $28 \%$ of cases (12). Subsequently, the FDA approved cabozantinib for the treatment of advanced MTC in November 2012.

In the absence of labeled drug, inclusion in a clinical trial should always be privileged for patients with refractory and progressive thyroid carcinoma. Unfortunately, in clinical practice, a substantial proportion of patients cannot be included in any trial for various reasons, such as the unavailability of a protocol or the presence of exclusion criteria, and this led to the off-label use of TKIs in referral French centers, part of the Tumeurs Réfractaires de la Thyroïde (TUTHYREF) network.

Targeted therapies are increasingly prescribed in patients with advanced or metastatic thyroid carcinoma, and only little is known about their efficacy according to organ-specific metastatic sites and in sequential treatment, as second- and third-line TKI therapy. This is indeed a major issue, because all TKIs used in these patients belong to the same family of anti-angiogenic drugs. We hereby report the efficacy of sorafenib, sunitinib, and vandetanib administered outside of clinical trials and we evaluated tumor response rate according to metastatic sites and sequential treatments in advanced thyroid cancer patients from five referral centers of the French TUTHYREF network.

\section{Subjects and methods}

\section{Patients}

All patients with metastatic or locally advanced DTC or advanced MTC who were treated with a TKI outside a clinical trial in one of the five TUTHYREF participating centers were retrospectively reviewed: these adult patients had at least one measurable lesion and had a baseline and at least one follow-up imaging study during TKI treatment in order to assess morphological tumor response. All patients provided informed consent and the study was approved by research ethics boards of the participating centers.

\section{Study endpoints}

The primary endpoints were tumor response rate and PFS in patients treated with TKIs administered outside of clinical trials. Secondary endpoints were tumor response rate and PFS with sequential TKI treatments (as secondand third-line therapies), and tumor response rate according to organ-specific metastatic sites. Only treatments given outside of clinical trials were analyzed for this study, but TKI treatments administered in the frame of clinical trials were considered while evaluating sequential TKI treatments, counting as first-line treatment. Doselimiting toxicities (DLTs), defined as requiring dose reduction or treatment withdrawal, were colligated and graded according to the US National Cancer Institute Common Terminology Criteria for Adverse Events V4.0.

\section{Morphological assessment}

Computed tomography (CT) scans were used to determine tumor responses during treatment with TKI, according to the Response Evaluation Criteria in Solid Tumors (RECIST) V1.1 (13). Target lesions (TLs) were defined as soft tissue metastatic lesions that could be measured in at least one dimension, with the longest diameter of at least $1 \mathrm{~cm}$ for most sites and $1.5 \mathrm{~cm}$ for lymph nodes. The soft tissue component of a bone lesion with a longest diameter of more than $1 \mathrm{~cm}$ could be considered as a TL and previously irradiated bone metastases were not considered as TLs. Other lesions that were less than these dimensions or that were not measurable were considered as non-TLs. 
All CT scans were reviewed by an independent radiologist (M Claude-Desroches) who was not aware of the outcome of any treatment. Tumor response was defined as progressive disease (PD) if there was $\geq 20 \%$ increase in the sum of the diameters of TLs or appearance of new metastatic lesions, as stable disease (SD) if there was a change between +19 and $-29 \%$ and PR was defined by a decrease of $\geq 30 \%$ in the sum of the diameters of TLs.

\section{Statistical analysis}

Quantitative data were expressed as means; qualitative data were expressed in percentages. Best tumor response was represented using a waterfall plot. PFS was defined as the time between TKI treatment initiation and the first subsequent event (progression or death from any cause). Patients who were alive and progression-free were censored at the date of their last follow-up visit. PFS was estimated using the Kaplan-Meier curve. Analyses were performed using SAS Statistical Software V9.1 (SAS Institute, Inc., Cary, NC, USA).

\section{Results}

\section{Clinical characteristics}

From November 2008 to December 2011, 62 patients (37 men (60\%), mean age: 61 years) with advanced thyroid cancer were treated with TKI outside of clinical trials and had available follow-up CT scan. All patients had distant metastases at baseline except one patient who had an unresectable locally advanced disease. Thyroid cancer histology was papillary in 22 patients (35\%), follicular (FTC) in five (8\%), oncocytic in five (5\%), poorly differentiated in 13 (21\%), and medullary in 17 (27\%). Referee pathologists of each TUTHYREF network region systematically reviewed all thyroid tumor histological slides. Among the 45 DTC patients, 41 had radioactive iodine (RAI) refractory tumors, defined by the absence of RAI uptake in one or more metastatic lesion(s), a disease progression within 16 months after a RAI treatment, or a persistent disease after a cumulative RAI dose of $\geq 600 \mathrm{mCi}$. Among the four DTC patients who were not treated with RAI, three had advanced poorly DTC, and one had a FTC with locally advanced and unresectable disease.

Thirty-three (53\%), 25 (40\%), and four (6\%) patients were treated with one, two, and three lines of TKIs respectively. Twenty-six patients had received previous TKI treatments in the frame of clinical trials (Supplementary Fig. 1, see section on supplementary data given at the end of this article and Supplementary Table 1), but only treatments given outside of clinical trials were analyzed for this study, totalizing 69 TKI treatments. Baseline clinical characteristics of patients and treatments administered are detailed in Tables 1 and 2 .

According to RECIST, 51 (82\%) patients had a PD over 12 months before treatment initiation, six (10\%) had PD over 14 months, and five (8\%) patients without demonstrated PD were treated with a TKI for large tumor burden or symptomatic disease.

\section{Therapy}

Among the 69 treatments administered outside of clinical trials, sorafenib was prescribed at a starting dose of $400 \mathrm{mg}$ twice a day in 39 patients and once a day in the other four

Table 1 Baseline clinical patient characteristics according to tumor histologic type.

\begin{tabular}{|c|c|c|}
\hline Characteristics & $n$ & Percentage $(\%)$ \\
\hline \multicolumn{3}{|l|}{ Gender } \\
\hline Male & 37 & 60 \\
\hline Female & 25 & 40 \\
\hline $\begin{array}{l}\text { Mean age (years), } \\
\text { mean (range), } n=62\end{array}$ & $61(27-77)$ & \\
\hline \multicolumn{3}{|l|}{ Histologic type } \\
\hline Papillary & 22 & 35 \\
\hline Follicular & 5 & 8 \\
\hline Hürthle cell & 5 & 8 \\
\hline Poorly differentiated & 13 & 21 \\
\hline Medullary & 17 & 27 \\
\hline \multicolumn{3}{|l|}{ Metastases at baseline } \\
\hline $\begin{array}{l}\text { No metastasis (locally } \\
\text { advanced) }\end{array}$ & 1 & 2 \\
\hline Metastases & 61 & 98 \\
\hline \multicolumn{3}{|l|}{ Sites of metastases at baseline } \\
\hline Lymph node & 46 & 74 \\
\hline Lung & 47 & 76 \\
\hline Bone & 28 & 45 \\
\hline Liver & 19 & 31 \\
\hline Pleura & 13 & 21 \\
\hline Adrenal & 6 & 10 \\
\hline \multicolumn{3}{|l|}{ Prior systemic treatments } \\
\hline Radioactive iodine & 38 & 61 \\
\hline Chemotherapy & 18 & 29 \\
\hline Radiotherapy & 40 & 65 \\
\hline $\begin{array}{l}\text { TKI (in the frame of clinical } \\
\text { trials) }\end{array}$ & 26 & 42 \\
\hline \multicolumn{3}{|l|}{ Number of TKI treatment lines } \\
\hline 1 & 33 & 53 \\
\hline 2 & 25 & 40 \\
\hline 3 & 4 & 6 \\
\hline$\geq 2$ & 29 & 47 \\
\hline
\end{tabular}

TKI, tyrosine kinase inhibitor; TNM, tumor, node, metastasis.

${ }^{a}$ Twenty-six TKI treatments previously received in the frame of clinical trials, including one sorafenib, three sunitinib, 18 vandetanib, two motesanib, one cabozantinib, and one lenvatinib treatments (Supplementary Fig. 1 and Supplementary table 1). Those treatments were not analyzed in this study. 
Table 2 TKI treatments administered outside of clinical trials, according to tumor histological type $(n=69)^{\mathrm{a}}$.

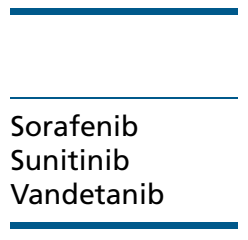

\begin{tabular}{c}
\hline Papillary \\
\hline 17 \\
6 \\
0 \\
\hline
\end{tabular}

\begin{tabular}{c}
\hline Follicular \\
\hline 5 \\
2 \\
0 \\
\hline
\end{tabular}

\begin{tabular}{c}
\hline Hürthle cell \\
\hline 4 \\
2 \\
0 \\
\hline
\end{tabular}

\begin{tabular}{c}
$\begin{array}{c}\text { Poorly } \\
\text { differentiated }\end{array}$ \\
\hline 13 \\
2 \\
0 \\
\hline
\end{tabular}

\begin{tabular}{c} 
Medullary \\
\hline 4 \\
3 \\
11 \\
\hline
\end{tabular}

ancluding exclusively TKI treatments received outside of clinical trials $(n=69)$ among the 62 patients, according to their tumor histological type.

patients. Sunitinib was prescribed at a starting dose of $37.5 \mathrm{mg}$ daily on a continuous basis for seven patients, for 3 weeks followed by 1 week off drug for seven patients, and for 4 weeks followed by 2 weeks off for one patient. Vandetanib was prescribed outside a clinical trial with nominative temporary treatment authorization at a starting dose of $300 \mathrm{mg}$ once a day in 11 MTC patients.

\section{Reasons for therapy outside of a clinical trial}

Patients were treated outside a clinical trial for one or several reasons: 26 patients had previously been treated with a TKI in a clinical trial, and this was an exclusion criterion for some further available therapeutic trials, two patients refused inclusion in a trial, and 12 had contra-indications for trial inclusion (previous cytotoxic chemotherapy, medical condition, absence of objective disease progression on CT scan, unavailability of a pre-treatment CT scan, and no previous RAI treatment). There was no protocol available at the time of treatment for 29 patients, one patient was not eligible for a rapidly progressing disease, in whom the possibility of receiving a placebo was considered medically unacceptable, and one patient was treated off-trial in another center and was then referred to one participating center.

\section{Morphological assessment}

The 340 CT scans for the assessment of treatment efficacy were done every 3 months, except for 13 CT scans that were done at an interval of 5-7 months.

Waterfall plots for best tumor response are shown on Fig. 1. Among the 39 DTC patients treated with sorafenib (median treatment duration: 7 months (3-28 months)), the PR rate was $15 \%$, SD for $\geq 6$ months: $38 \%$, and median PFS: 7.2 months. Among the 12 DTC patients treated with sunitinib (median treatment duration: 6 months (3-20 months)), PR rate was $8 \%$, SD for $\geq 6$ months: $33 \%$, and median PFS: 6.5 months. Of the 11 MTC patients treated with vandetanib (median treatment duration: 7 months
(4-14 months)), PR rate was $36 \%$, SD for $\geq 6$ months: $27 \%$, and median PFS was not reached.

Best mean tumor response rates according to metastatic sites are detailed in Table 3. In DTC patients, lung metastases were the most responsive lesions to treatment and bone and pleural lesions were the most refractory lesions. In MTC patients, liver and lymph node metastases were the most responsive lesions and bone lesions were the most refractory lesions to treatment. There was no pleural lesion and only a few lung $(n=2)$ and bone $(n=2)$ metastases analyzed in these patients.

In first-line sorafenib or sunitinib therapy in 24 DTC patients, PR rate was $29 \%$, but with second-line $(n=24)$ and third-line $(n=3)$ treatments there was no PR $(P=0.02)$. However, median PFS was similar in the second-line compared with the first-line treatment (6.7 vs 7.0 months, $\mathrm{HR}=0.96$ (95\% CI 0.53-1.74), $P=0.90$; Table 4). We could not evaluate vandetanib efficacy in sequential treatment because only two patients received it as a second-line therapy.

\section{Toxicities and complications}

Among the 39 DTC patients treated with sorafenib, 26 experienced DLTs (46 events: 34 dose reductions, six temporary, and six definitive withdrawals). The most frequent were hand-foot syndrome $(28 \%)$, weight loss (26\%), diarrhea (23\%), fatigue (10\%), and stomatitis (10\%) (grades 2 and 3). Of the 12 DTC patients treated with sunitinib, six had DLTs (ten events: seven dose reductions and three temporary withdrawals), the most frequent being fatigue (25\%), diarrhea (17\%) and anorexia (17\%) (grades 2 and 3). One grade 4 proteinuria with nephrotic syndrome occurred during sunitinib treatment at a $37.5 \mathrm{mg} / \mathrm{day}, 3 / 4$ weeks, but kidney function recovered after drug withdrawal, and sunitinib could be reintroduced 1 month later at a lower dosage $(25 \mathrm{mg} /$ day, $3 / 4$ weeks). Among the 11 MTC patients treated with vandetanib, seven had DLTs (11 events: eight dose reductions and three temporary withdrawals), the most 


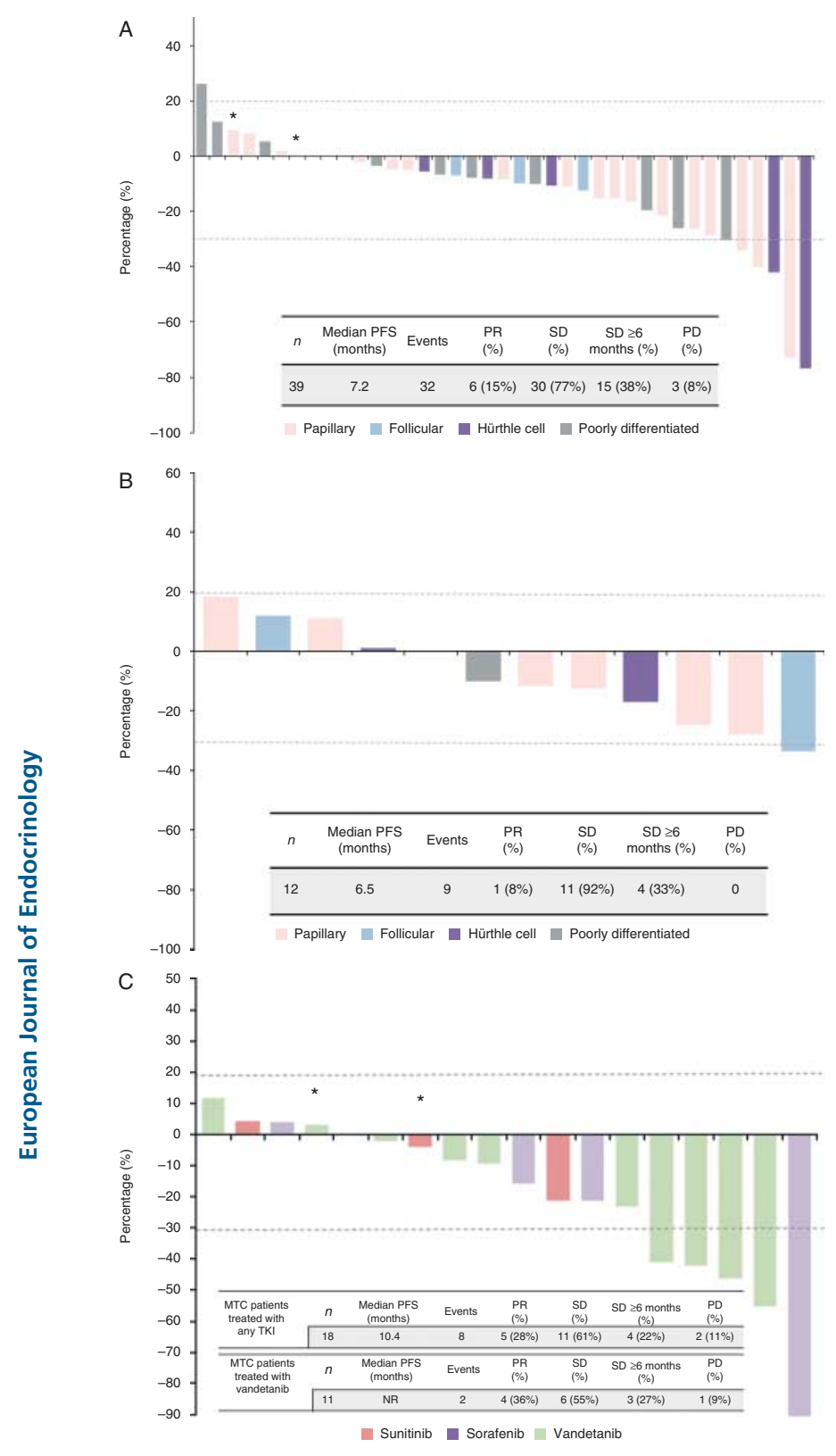

\section{Figure 1}

Waterfall plots of best tumor response in target lesions (TLs; $\%$ of change from baseline), median PFS and tumor response (RECIST V1.1) in patients with refractory thyroid carcinoma treated with TKIs outside of clinical trials. (A) DTC patients treated with sorafenib; (B) DTC patients treated with sunitinib; and (C) MTC patients treated with any TKI. *Patients with PD as best tumor response in non TLs but with SD in TLs. DTC, differentiated thyroid carcinoma; MTC, medullary thyroid carcinoma; PFS, progression-free survival; NR, not reached; $\mathrm{PD}$, progressive disease; PR, partial response; SD, stable disease; TKI, tyrosine kinase inhibitor. frequent being corrected QT (QTc) interval prolongation grade 3 (>500 ms) in $27 \%$. No Torsade de pointes or cardiac arrhythmia occurred, and QTc interval normalized after vandetanib withdrawal or dose reduction.

Among the six patients who were not eligible for a clinical trial because of a medical condition (left bundle branch block on electrocardiogram, Gilbert's syndrome, epilepsy, concomitant renal cell carcinoma, and anticoagulation for two patients), no complication linked to any of these conditions occurred.

\section{Discussion}

The main goal of this study was to evaluate sorafenib, sunitinib, and vandetanib treatments in a 'real life' clinical setting, outside of clinical trials, in patients with metastatic or locally advanced thyroid carcinomas. Indeed, in clinical practice, a substantial proportion of patients cannot be included in any trial for various reasons; nonetheless, these patients need a treatment, because their disease will cause significant symptoms and even death. As secondary endpoints, we analyzed tumor response according to metastatic sites and sequential TKI treatments.

All CT scans were reviewed by an independent radiologist who was not aware of the outcome of any treatment. Thirteen CT scans out of 340 were done at an interval of $>3$ months and this is related to the retrospective and non-trial nature of our study. Among these 13 CT scans, only three were the last patients' scans analyzed for tumor response, done at a 5-month interval, and supplemental analyses confirmed that there was no impact on study results, as they represent a very small proportion of the total of analyzed scans and patients.

DTC patients treated with sorafenib or sunitinib had shorter median PFS than those of previous publications respectively 7.2 vs $10.8-19.8$ months $(4,5,8,14)$ and 6.5 vs 12.8-19 months $(6,14)$. These differences may be related to the great heterogeneity of patients among trials. In our study, the only exclusion criterion was the unavailability of CT scans to assess tumor response, and patients could have received prior therapy, including a TKI. The vast majority of our patients were progressive before treatment, according to RECIST, and the few who were not progressive had symptoms and a large tumor burden. Furthermore, we included all subtypes of DTCs, in particular poorly differentiated carcinomas, which are known to be aggressive tumors, representing 21\% of our cohort. So our patients differ from those previously studied and represent more precisely the population 
Table 3 Best mean tumor response in DTC and MTC patients according to organ-specific metastatic sites.

\begin{tabular}{|c|c|c|c|c|c|c|}
\hline \multirow[b]{2}{*}{ Metastatic site } & \multicolumn{3}{|c|}{ DTC patients $^{a}$} & \multicolumn{3}{|c|}{ MTC patients ${ }^{\mathrm{b}}$} \\
\hline & $n$ & Percentage (\%) & Range (\%) & $n$ & Percentage (\%) & Range (\%) \\
\hline Liver & 6 & -18 & -32 to -11 & 8 & -30 & -100 to -4 \\
\hline Lung & 41 & -23 & -100 to +20 & 2 & -19 & -27 to -11 \\
\hline Lymph node & 32 & -13 & -73 to +40 & 15 & -29 & -70 to +12 \\
\hline Pleura & 7 & -5 & -35 to +11 & 0 & NA & NA \\
\hline Bone & 12 & -5 & -25 to +15 & 2 & -8 & -15 to 0 \\
\hline
\end{tabular}

DTC, differentiated thyroid carcinoma; MTC, medullary thyroid carcinoma; NA, not applicable.

${ }^{a}$ DTC patients treated with sorafenib and sunitinib outside of clinical trials.

${ }^{\mathrm{b}}$ MTC patients treated with vandetanib, sorafenib, and sunitinib outside of clinical trials.

whose physicians have to manage while prescribing TKIs outside of clinical trials.

Adverse events were similar to those previously reported with sunitinib and sorafenib. One grade 4 proteinuria with nephrotic syndrome and kidney failure occurred with sunitinib, which recovered following drug withdrawal. Nephrotic syndrome and severe kidney dysfunction, related to anti-vascular endothelial growth factor receptor, are rare with sunitinib (15).

Even if median PFS was not reached and that median treatment duration was relatively short in MTC patients treated with vandetanib, a considerable PR rate was observed, comparable to that observed in a previous study (11). This result reinforces vandetanib treatment efficacy in MTC patients. The most common adverse event was the QTc interval prolongation which normalized after temporary withdrawal, no related cardiac complication was observed, and vandetanib treatment could be safely resumed at lower doses.

No related complication occurred in patients excluded from clinical trials for medical conditions, such as anticoagulation or significant anomaly on electrocardiogram. In our opinion, this is an important finding that retrospectively justifies TKI treatment outside of clinical trials, because these patients, who would not have been treated otherwise because of their exclusion criteria, had a clear indication for treatment and could safely benefit from the therapy. Of course, risks and benefits need to be carefully weighed in these patients and the decision to initiate a TKI treatment should always be taken by a tumor board of experienced physicians.

Evaluation of TKIs' efficacy in sequential treatment showed that DTC patients treated with second-line therapy (sorafenib or sunitinib) had SD as best response without any PR, but had a similar median PFS compared with first-line treatment, supporting a clinical benefit for second-line TKI therapy. Similarly, in renal cell cancer, the efficacy of second-line sunitinib treatment was close to that of first-line therapy, suggesting limited crossresistance (16), and comparable PR rates were shown with cabozantinib and lenvatinib in both DTCs and MTCs in first- and second-line treatment $(12,17,18,19)$.

Table 4 Median PFS and tumor response (RECIST V1.1) according to sequential treatments with sorafenib and sunitinib in DTC patients.

\begin{tabular}{|c|c|c|c|c|c|}
\hline Treatment & Line of treatment & $n$ & Events & $\begin{array}{c}\text { Median PFS } \\
\text { (months) }\end{array}$ & $\mathbf{P R}(\%)$ \\
\hline \multirow[t]{3}{*}{ Sorafenib } & In first-line treatment & 20 & 15 & 7.2 & 30 \\
\hline & In second-line treatment & 19 & 17 & 6.7 & 0 \\
\hline & In third-line treatment & 0 & NA & NA & NA \\
\hline \multicolumn{2}{|c|}{ Median PFS comparison: first-line vs second-line treatments ${ }^{a}$} & \multicolumn{4}{|c|}{$\mathrm{HR}=0.79(95 \% \mathrm{Cl} 0.40-1.56), P=0.5$} \\
\hline \multirow[t]{4}{*}{ Sunitinib } & In first-line treatment & 4 & 3 & 6.7 & 25 \\
\hline & In second-line treatment & 5 & 3 & 13.7 & 0 \\
\hline & In third-line treatment & 3 & 3 & 4.5 & 0 \\
\hline & & \multicolumn{4}{|c|}{$\mathrm{HR}=0.55(95 \% \mathrm{Cl} 0.14-2.23), P=0.4$} \\
\hline \multirow{4}{*}{ Sunitinib or sorafenib } & In first-line treatment & 24 & 18 & 7.0 & 29 \\
\hline & In second-line treatment & 24 & 20 & 6.7 & 0 \\
\hline & In third-line treatment & 3 & 3 & 4.5 & 0 \\
\hline & & \multicolumn{4}{|c|}{$\mathrm{HR}=0.96(95 \% \mathrm{Cl} 0.53-1.74), P=0.9$} \\
\hline
\end{tabular}

DTC, differentiated thyroid carcinoma; PFS, progression-free survival; PR, partial response; NA, not applicable; TKI, tyrosine kinase inhibitor. ${ }^{a}$ Median PFS comparison with log-rank test and tumor response comparison with Fischer test between first-line treatment and second-line treatment. 
This analysis was limited by the small number of DTC patients treated with sunitinib: four patients in first-line treatment and five patients in second-line treatment. Therefore, we cannot recommend any specific treatment sequence (sorafenib and then sunitinib, or sunitinib and then sorafenib) and a further prospective study should be conducted to better evaluate this issue. However, 20 DTC patients were treated with sorafenib in first-line therapy and 19 in second-line treatment, and our results suggest that sorafenib is still active in second-line therapy. Finally, because of the small number of patients in second-line treatment, we could not identify predictive factors of prolonged PFS in second-line setting.

Bone metastases from thyroid carcinoma, which are known to be poorly responsive to radioiodine therapy, also seem to be more frequently refractory to TKI treatments $(14,20)$. One French retrospective study showed that $35 \%$ of thyroid cancer patients with response in soft tissue lesions had bone progression on TKI treatment (21). Furthermore, in a study with DTC patients treated with sorafenib or sunitinib, all pleural and bone metastases were progressive, while a median tumor response of $-22 \%$ was observed in lung metastases (14). These results are consistent with our findings and imply that loco-regional treatment modalities should be considered for progressive or symptomatic bone lesions prior or even during a TKI treatment, which may allow to maintain the treatment in patients with responses in soft tissue lesions.

\section{Conclusion}

This is the largest study evaluating sunitinib, sorafenib, and vandetanib therapies outside of clinical trials and the first to evaluate sequential treatments in DTCs. Nevertheless, it is important to emphasize that patients with advanced thyroid carcinoma should always be enrolled, whenever possible, in clinical trials. Only exclusion criteria or unavailability of any protocol may lead to TKI prescription outside of clinical trials in referral centers, as they will help to control patients' disease, even in second-line treatment. Indeed, DTC patients treated with second-line TKI therapy had SD as best response without PR, but had a similar median PFS compared with first-line treatment.

Bone and pleural metastases were the most refractory to treatment. The physiopathology of this variable tissue response remains unknown $(20,22)$ and therapeutic strategies need to be set up to overcome this significant limitation to treatment.

Finally, our study's limitations are the small number of patients and its retrospective nature. Larger prospective trials could answer many important questions, such as recommending a specific sequence in TKI therapy and identifying predictive factors of prolonged PFS in secondline treatment.

Supplementary data

This is linked to the online version of the paper at http://dx.doi.org/10.1530/ EJE-13-0825.

Declaration of interest

M Brassard, M Schlumberger, S Leboulleux, and C de la Fouchardière have attended advisory boards for Astra Zeneca. M Schlumberger and $\mathrm{M}-\mathrm{H}$ Massicotte have received honoraria from Astra Zeneca and Bayer, and M Schlumberger received research funding from Astra Zeneca, Amgen, Exelixis, Bayer, and Eisai. All remaining authors have declared no conflicts of interest.

\section{Funding}

This research did not receive any specific grant from any funding agency in the public, commercial or not-for-profit sector.

\section{Author contribution statement}

M-H Massicotte and M Brassard collected data, contributed to study design, literature search, figures elaboration, data analysis and interpretation, and writing of the report. M Claude-Desroches collected data, contributed to study design, data analysis, writing of the report, and revised all CT scans of this study. I Borget contributed to study design, data analysis and interpretation, figures elaboration, and writing of the report. $F$ Bonichon was involved in management of patients, study design, literature search, data collection, data analysis, and review of the report. A-L Giraudet, C Do Cao, and C N Chougnet contributed to management of patients, data collection, and review of the report. S Leboulleux contributed to study design, data analysis, management of patients, and review of the report. E Baudin was involved in data collection, management of patients, and review of the report. $\mathrm{M}$ Schlumberger and $\mathrm{C}$ de la Fouchardière contributed to management of patients, literature search, study design, data interpretation, and review of the final report.

\section{Acknowledgements}

This study has been presented as an oral presentation at the American Thyroid Association (ATA) annual meeting, Quebec, Canada, 2012 and as a poster presentation at the American Society of Clinical Oncology (ASCO) annual meeting, Chicago, United States, 2013.

\section{References}

1 Durante C, Haddy N, Baudin E, Leboulleux S, Hartl D, Travagli JP, Caillou B, Ricard M, Lumbroso JD, DeVathaire F et al. Long term outcome of 444 patients with distant metastases from papillary and 
follicular thyroid carcinoma: benefits and limits of radioiodine therapy. Journal of Clinical Endocrinology and Metabolism 200691 2892-2899. (doi:10.1210/jc.2005-2838)

2 Chougnet C, Brassard M, Leboulleux S, Baudin E \& Schlumberger M. Molecular targeted therapies for patients with refractory thyroid cancer. Clinical Oncology 201022 448-455. (doi:10.1016/j.clon.2010.04.008)

3 De la Fouchardière $\mathrm{C} \&$ Droz JP. Targeted therapies and thyroid cancer: an update. Anti-Cancer Drugs 201122 688-699. (doi:10.1097/CAD. 0b013e32834319c7)

4 Gupta-Abramson V, Troxel AB, Nellore A, Puttaswamy K, Redlinger M, Ransone K, Mandel SJ, Flaherty KT, Loevner LA, O'Dwyer PJ et al. Phase II trial of sorafenib in advanced thyroid cancer. Journal of Clinical Oncology 200826 4714-4719. (doi:10.1200/JCO.2008.16.3279)

5 Kloos RT, Ringel MD, Knopp MV, Hall NC, King M, Stevens R, Liang J, Wakely PE Jr, Vasko VV, Saji M et al. Phase II trial of sorafenib in metastatic thyroid cancer. Journal of Clinical Oncology 200927 1675-1684. (doi:10.1200/JCO.2008.18.2717)

6 Carr LL, Mankoff DA, Goulart BH, Eaton KD, Capell PT, Kell EM, Bauman JE \& Martins RG. Phase II study of daily sunitinib in FDG-PETpositive, iodine-refractory differentiated thyroid cancer and metastatic medullary carcinoma of the thyroid with functional imaging correlation. Clinical Cancer Research 201016 5260-5268. (doi:10.1158/ 1078-0432.CCR-10-0994)

7 Cohen EE, Needles BM, Cullen KJ, Wong SJ, Wade JL, Ivy SP, Villaflor VM, Seiwert TY, Nichols K \& Vokes EE. Phase 2 study of sunitinib in refractory thyroid cancer. Journal of Clinical Oncology 2008 26 (Suppl) 6025 (abstract).

8 Brose MS, Nutting C, Jarzab B, Elisei R, Siena S, Bastholt L, de la Fouchardiere C, Pacini F, Paschke R, Shong YK, Sherman SI et al. Sorafenib in locally advanced or metastatic patients with radioactive iodine-refractory differentiated thyroid cancer: the phase 3 DECISION trial. Journal of Clinical Oncology $2013 \mathbf{3 1}$ (Suppl) 4 (abstract).

9 Leboulleux S, Bastholt L, Krause T, de la Fouchardiere C, Tennvall J, Awada A, Gómez JM, Bonichon F, Leenhardt L, Soufflet C et al. Vandetanib in locally advanced or metastatic differentiated thyroid cancer: a randomised, double-blind, phase 2 trial. Lancet Oncology 2012 13 897-905. (doi:10.1016/S1470-2045(12)70335-2)

10 Lam ET, Ringel MD, Kloos RT, Prior TW, Knopp MV, Liang J, Sammet S, Hall NC, Wakely PE Jr, Vasko VV et al. Phase II clinical trial of sorafenib in metastatic medullary thyroid cancer. Journal of Clinical Oncology 2010 28 2323-2330. (doi:10.1200/JCO.2009.25.0068)

11 Wells SA Jr, Robinson BG, Gagel RF, Dralle H, Fagin JA, Santoro M, Baudin E, Elisei R, Jarzab B, Vasselli JR et al. Vandetanib in patients with locally advanced or metastatic medullary thyroid cancer: a randomized, double-blind phase III trial. Journal of Clinical Oncology 201230 134-141. (doi:10.1200/JCO.2011.35.5040)

12 Elisei R, Schlumberger MJ, Müller S, Schöffski P, Brose MS, Shah MH, Licitra L, Jarzab B, Medvedev V, Kreissl MC et al. Cabozantinib in progressive medullary thyroid carcinoma. Journal of Clinical Oncology, 2013.
13 Eisenhauer EA, Therasse P, Bogaerts J, Schwartz LH, Sargent D, Ford R, Dancey J, Arbuck S, Gwyther S, Mooney M et al. New response evaluation criteria in solid tumours: revised RECIST guideline (version 1.1). European Journal of Cancer 200945 228-247. (doi:10.1016/ j.ejca.2008.10.026)

14 Cabanillas ME, Waguespack SG, Bronstein Y, Williams MD, Feng L, Hernandez M, Lopez A, Sherman SI \& Busaidy NL. Treatment with tyrosine kinase inhibitors for patients with differentiated thyroid cancer: the M. D. Anderson experience. Journal of Clinical Endocrinology and Metabolism 201095 2588-2595. (doi:10.1210/ jc.2009-1923)

15 Takahashi D, Nagahama K, Tsuura Y, Tanaka H \& Tamura T. Sunitinibinduced nephrotic syndrome and irreversible renal dysfunction. Clinical and Experimental Nephrology 201216 310-315. (doi:10.1007/ s10157-011-0543-9)

16 Zimmermann K, Schmittel A, Steiner U, Asemissen AM, Knoedler M, Thiel E, Miller K \& Keilholz U. Sunitinib treatment for patients with advanced clear-cell renal-cell carcinoma after progression on sorafenib. Oncology 200976 350-354. (doi:10.1159/000209961)

17 Cabanillas ME, Brose MS, Ramies DA, Lee Y, Miles D \& Sherman SI. Antitumor activity of cabozantinib (XL184) in a cohort of patients (PTS) with differentiated thyroid cancer (DTC). Journal of Clinical Oncology 201230 (Suppl) 5547 (abstract).

18 Schlumberger M, Jarzab B, Cabanillas ME, Robinson B, Pacini F, Ball DW, McCaffrey JC, Newbold K, Allison R, Martins R et al. A phase II trial of the multitargeted kinase inhibitor lenvatinib (E7080) in advanced medullary thyroid cancer (MTC). Journal of Clinical Oncology 201230 (Suppl) 5591 (abstract).

19 Sherman SI, Jarzab B, Cabanillas ME, Licitra LF, Pacini F, Martins R, Robinson B, Ball D, McCaffrey J, Shah MH et al. A phase II trial of the multi-targeted kinase inhibitor, lenvatinib (E7080), in advanced radioiodine-refractory differentiated thyroid cancer (DTC). Journal of Clinical Oncology 201129 (Suppl) 5503 (abstract).

20 Hoftijzer H, Heemstra KA, Morreau H, Stokkel MP, Corssmit EP, Gelderblom H, Weijers K, Pereira AM, Huijberts M, Kapiteijn E et al. Beneficial effects of sorafenib on tumor progression, but not on radioiodine uptake, in patients with differentiated thyroid carcinoma. European Journal of Endocrinology 2009161 923-931. (doi:10.1530/ EJE-09-0702)

21 Haissaguerre M, Leboulleux S, Bonichon F, Borget I, Chougnet CN, Al Ghuzlan A, Hartl DM, Mirghani H, Deandreis D, Baudin E et al. Impact of molecular targeted therapies in patients with bone metastases of differentiated thyroid carcinoma. Thyroid $2012 \mathbf{2 2}$ (Suppl 1) oral 9 (abstract).

22 Cabanillas ME, Hu MI, Durand JB \& Busaidy NL. Challenges associated with tyrosine kinase inhibitor therapy for metastatic thyroid cancer. Journal of Thyroid Research 2011 2011. Artice ID 985780. (doi:10.4061/ 2011/985780)

Received 8 October 2013

Revised version received 17 December 2013 Accepted 14 January 2014 